\title{
DŎMUS : un mot-relique dans la toponymie de la France
}

\author{
Pierre-Henri Billy \\ Université Paris-I Sorbonne, CNRS, UMR 8589, LAMOP \\ billy@vjf.cnrs.fr
}

Résumé: Latin domus n'était jusqu'ici connu en France que dans le lexique latin médiéval et la vie française de saint Léger. La (micro)toponymie permet de restituer sa présence en de nombreux exemplaires, sous diverses formes morphologiques ; les sources narratives et normatives permettent de restituer son évolution sémantique.

Mots-clefs: domus ; Moyen Âge ; France ; sémantique ; toponymie.

\begin{abstract}
In France, Latin domus was known up to now only in the medieval Latin lexicon and the French life of st. Léger. Place names reveal its presence in many items and various morphological forms ; the narrative and normative sources allow to restore its semantic evolution.
\end{abstract}

Keywords: domus ; Moyen Âge ; France ; sémantique ; toponymie. 

La singularité des mots-reliques est d'être trouvés par le seul hasard des lectures ou des recherches, puis confortés dans leur statut par une collection d'exemples susceptible d'écarter toute erreur d'interprétation. Le présent article est le fruit d'une rencontre improbable dans la quête de mots-reliques latins pouvant figurer dans les cartulaires bretons.

Le latin DŎMUS est réputé n'avoir eu dans le domaine galloroman qu'un descendant lexical, dom, employé dans la Chanson de saint Léger datée des environs de l'an mil (FEW III, 135b). Le manuscrit, transcrit probablement au début du $\mathrm{XI}^{\mathrm{e}}$ s., reproduit un original du siècle précédent : si la retranscription a été exécutée en Poitou, certains traits phonétiques pourraient relever aussi bien du wallon que du bourbonnais (Hilty, 1997: 73), confortant ainsi la thèse de l'intervention du scriptorium d'Ébreuil (De Poerck, 1963: 14-15), ce qui en fait une œuvre historiquement et linguistiquement localisée à la charnière des domaines galloromans. Le lexème ne réapparaît pas plus tard, ni en français, ni en occitan.

La strophe 33 du poème dédié à la vie de saint Léger rapporte : Cil Lodeberz furet buons huom,/ Et sant Ledgier duist a son duom./ "Chlodebert était un homme bon, et il ramena saint Léger à sa maison". Bien que Wartburg traduise duom par "maison", on est assuré qu'il ne s'agit pas d'une (simple) maison, mais bien du palais épiscopal d'Autun, construit en pierres contrairement aux habitats les plus fréquents de l'époque. D'autre part, la Passio sancti Leodegarii d'Ursinus qui sert de base au texte rapporte ainsi l'événement : Acceptum eumdem cum ad suam domum deduceret (Paris, 1872: 315). L'auteur du poème, qui a conscience que domus signifie "palais épiscopal", ne trouvant pas de traduction possible en français, crée donc cet hapax savant dom, mais au masculin contrairement à son original latin et très probablement pour la seule rime : déjà, quatre siècles plus tôt, Grégoire de Tours employait domus ecclesiae pour désigner le siège de l'évêque (pergit ad domum ecclesiae per tenebras noctis (Krusch \& Lewison, 1951: Hist. I, 44)).

D'après Wartburg, DŎMUS a eu en revanche des résultats romans en Italie : domo "maison" en logudorien et campidanien, dom "domicile" en ancien bergamasque, domo "maison" en ancien lombard ; ainsi qu'en ancien rhétoroman car les Gloses de Cassel, rédigées au VIII s., l'utilisent : domo cadam (Marchot, 1895: 11), dont la forme apparemment déclinée cèle en fait la prononciation orale et révèle en outre, comme ses synonymes italiens plus tardifs, le genre masculin. De son 
côté, Meyer-Lübke ajoute la forme logudorienne domoz "possession féodale" (1935: 246), qui ne figurait toujours pas dans sa seconde édition datée de 1911.

Ces résultats lexicaux sont, toujours d'après Wartburg, à l'origine de noms de villages du type Domo dans les Marches, la Lombardie, le Piémont et la Toscane : sur latin médiéval domus "matrice d'une paroisse ; paroisse", lexème masculin, d'où italien domo "habitation" dès le $\mathrm{XII}^{\mathrm{e}} \mathrm{s}$. Ajoutons des noms calabrais comme Domus de Maria et Domusnovas, formés sur le pluriel de domu qui désigne le centre d'un habitat rural complexe regroupant des bâtiments d'habitation et d'exploitation entourés de leurs terres, ce qui représente le schéma de l'antique villa (Gasca Queirazza et alii, 2006: 295). Ajoutons enfin des noms sardes comme Domor Novas, formé sur le pluriel du sarde domo "maison" (Wolf, 1998: 147) : le lexème est féminin, cf. domo "lieu de résidence ; maison" au XIII ${ }^{\mathrm{e}}$ s. et "maison de Dieu" au XIV e s., d'où le genre du toponyme.

La Catalogne possède aussi de rares témoins toponymiques de DŎMUS, mais au féminin : La Doma à La Garriga (Vallès Oriental), et son pluriel Les Domes à Vallfogona de Ripollès (Ripollès), avec des homonymes sur l'île d'Ibiza et dans le Baix Empordà (Coromines, 1995: 21). Si Les Domes désigne un hameau sans église, La Doma est le nom de l'ancienne église située en dehors du village de La Garriga. En catalan, le féminin doma désigne une charge ecclésiastique, existant dans quelques églises cathédrales ou canoniales, assumée alternativement par semaines : son étymologie est clairement latin HEBDŎMĂDA "semaine". Cet argument suffit à Coromines (1995: 21) pour rendre compte de l'étymologie de La Doma de La Garriga ; mais il ne se pose pas la question de savoir si cette église est cathédrale ou canoniale - ce qu'elle n'est pas -, ni si les autres noms de lieux catalans désigneraient aussi une église cathédrale ou canoniale. Cette absence de particularité, de même que l'existence de formes plurielles telles Les Domes, permettent de plaider ici pour des résultats de DŎMUS : au sens ecclésiastique du terme (domus Dei "église") pour l'église dite La Doma, au sens habitationnel du terme (domus "maison") pour le village dit Les Domes.

L'évolution sémantique de DŎMUS est relativement classique. Contrairement au grec $\delta o ́ \mu o \varsigma$ qui désigne un bâtiment construit, le latin désigne d'abord la famille qui habite ledit bâtiment, à tel point que les 
formes déclinées domum / domi / domo signifient "chez soi" (Hummel, 2004: 56). Les textes latins de l'époque dite classique exploreront toutes les facettes du bâtiment : "demeure", "maison (urbaine)", "pays", "personnel dépendant d'une maison" (Glare, 2012: 628). Le premier diminutif attesté dans ce sens figure au $\mathrm{I}^{\mathrm{er}}$ s. a.J.-C. chez l'architecte Vitruve : domuncula "petite maison" (ibid.). Le second diminutif, domumcella "petite maison", est attesté à la fin du IV ou au début du Ve s. dans la Peregrinatio Aetheriae (récit de pèlerinage en Terre sainte : XXXVI, 5) et sous la forme domucella vers 570 dans les Papyri édités par Marini (Souter, 1949: 112). Et c'est désormais ce champ sémantique seul qui se retrouvera dans les textes latins de quelque époque qu'ils soient, même si nombre d'auteurs chrétiens l'emploieront au sens figuré.

Autour de l'an 400, saint Jérôme emploie dans la Vulgate le mot domus pour traduire les notions de "demeure", "maison fortifiée, tour", "temple (de Jérusalem)". Quant à domus Dei, la locution désigne une église dans les Actes du Concile de Carthage en 411, un cloître chez saint Benoît un siècle plus tard (Blaise, 1954: 292).

À la fin du $\mathrm{VI}^{\mathrm{e}}$ s., Grégoire de Tours utilise domus dans deux acceptions bien différentes mais que seul un esprit moderne saurait, à tort, opposer : au sens de "maison (d'habitation)" à l'intérieur d'une villa, comme dans la phrase : Nam sepe a novo fundans villas, ponens vinias, aedificans domus, culturas eregens (Krusch \& Lewison, 1951: Hist. VI, 20) ; au sens de "village", en parfait synonyme de villa, comme dans la phrase : in rure domus Sexciacensis, quod in eius possessione subsistebat, eclesiam aedificavit; exinde iterum in alia villa aliud aedificavit templum (Krusch, 1885a: Glor. conf. 49). Ce dernier texte doit aussi être comparé avec le paragraphe précédent (Krusch, 1885a: Glor. conf. 48) : Infra terminum autem Beorretanae urbis in vico Sexciacensi sanctus Iustinus presbiter quiescit. Grégoire de Tours met donc sur un même plan sémantique domus, villa et vicus qui sont employés tous trois pour désigner le village appelé Sexciacus. Pour sa part, M. Heinzelmann interprète ici domus comme désignant une partie d'un vicus et donc une circonscription administrative dépendant de ce vicus (Heinzelmann, 1993: 61). Le texte est pourtant clair : le vicus est partie intégrante de la circonscription administrative ici appelée Beorretana urbs, et Grégoire emploie domus et vicus dans un seul et même sens, celui de villa "village". Parmi les autres acceptions, citons celle-ci : in orato- 
rio Marciacensis domus, Arverni territurii, eius reliquiae contenentur (Krusch, 1885b: Glor. mart. I, 8), où domus désigne sans ambiguïté un monastère, en l'occurrence celui de Marsat (Puy-de-Dôme).

Dans la seule Bretagne, domus, outre son emploi fréquent au sens de "maison d'habitation", rurale ou urbaine, dans le Cartulaire de Redon, est employé dans les Gesta sanctorum Rotonensium, rédigés entre 868 et probablement avant 900, pour désigner une habitation (paysanne) en bois transportée sur des charrettes à bœuf pour être remontée (domum suam ex tabulis ligneis fabricatam) ; dans d'autres sources carolingiennes pour désigner une demeure aristocratique (Jones, 2001: 46). Le palais du prince Ausoch (domum Ausochi) dans la Vita sancti Iudicaelis rédigée au $\mathrm{XI}^{\mathrm{e}}$ s. par Ingomar (Fawtier, 1925: 188), le palais de la reine de Brest au XI ${ }^{\mathrm{e}}$ s. (Oheix, 1913: 34). Aussi un château seigneurial, manoir ou non, au XII ${ }^{\mathrm{e}}$ s. en Haute-Bretagne (Meuret, 1993: 375).

Toujours en Bretagne, comme ailleurs, d'autres sens apparaissent plus tard ou survivent : "prieuré" au XI ${ }^{\mathrm{e}}$ s. (Le Men \& Ernault, 1886: n53) ; "grange dîmière" (Morice, 1742: I, col.1141) ; "marché couvert, cohue" (La Borderie, 1885: nLXXVIII) ; "fortification" en 1223 (Rosenzweig, 1895: n²59). Maisons templières, hospitalières, léproseries, églises priorales ou abbatiales, cathédrales etc. sont ainsi désignées par domus.

Si domus est du genre féminin en latin classique et, semble-t-il, impérial, il peut passer au masculin dans un certain nombre d'occurrences : les sources qui les contiennent montrent toujours une réelle variabilité du genre, et au profit du féminin en terme de fréquence.

Le premier texte dans lequel apparaît le genre masculin est la Vita Eligii Noviomagensis, rédigée en 634 (Krusch, 1902: II, 68). Puis au $\mathrm{VIII}^{\mathrm{e}}$ s. : la Vita Wilfridi episcopi Eboracensis rédigée par Eddius Stephanus vers 710-20 (Krusch \& Levison, 1913: 262) ; le Lucae evangelium expositio de Bède rédigé vers 720 (Migne, 1850b: XCII, col.541), les In Pentateuchum Commentarii attribués à Bède (Migne, 1850a: XCI, col.390); le capitulaire de Paderborn en 785 (Pertz, 1835: $\left.n^{\circ} 26\right)$. Ensuite au IX $X^{e}$ s. : la Lex Salica vers 802-03 (Eckhardt, 1969: 80) ; une charte d'échange entre l'archevêque d'Arles et le comte Leibulfe, en 824 (Albanès \& Chevalier, 1901: $\left.{ }^{\circ} 195\right)$; le De divortio Lotharii regis rédigé par Hincmar de Reims en 860 (Böhringer, 1992: 185). À partir du $\mathrm{X}^{\mathrm{e}}$ s., les chartes deviennent majoritaires parmi les sources qui attestent le masculin, notamment en Catalogne : cet emploi se fera de plus 
en plus fréquent à partir du XII ${ }^{\mathrm{e}} \mathrm{s}$. L'apparition du masculin dans des documents originaux ne laisse aucun doute sur leur caractère originel.

Dans la toponymie, la conservation du genre féminin se marquera par le changement de déclinaison : domus $>$ doma.

Le nombre même de domus est sujet à variation pour désigner pourtant non seulement le même objet, mais un seul. C'est l'exemple offert par la vita prima de saint Samson de Dol, rédigée au milieu du VIII ${ }^{\mathrm{e}} \mathrm{s}$. : subitaneum ac dirum incendium invasit domum [...]. Emenda, quesumus, domos tuas, quo non hac ustione consumantur (Flobert, 2002: II, 15). L'objet ainsi désigné est le monastère du saint homme : l'on pourra arguer que, dans le premier cas, le singulier désigne soit l'ensemble de l'entité monastère, soit le seul bâtiment ecclésial, que dans le second il désigne tous les bâtiments inclus dans ladite entité, à savoir, pour l'essentiel, l'église et les bâtiments conventuels. Rappelons que domus pouvait désigner, à cette époque et depuis la première littérature chrétienne (Mohrmann, 1962: 163-164), aussi bien la seule église ou oratoire que l'ensemble conventuel (cf. l'exemple de Grégoire de Tours, cité ci-dessus). Dans le texte, l'ecclesia est clairement séparée de la domus en chaume : singulier et pluriel sont employés pour désigner le seul bâtiment que l'auteur décrit en flammes, mais le singulier dans le récit même, le pluriel dans une supplique au style direct. Le pluriel n'aurait donc ici de valeur qu'emphatique. Dans le Quercy, mais bien plus tard, l'usage du pluriel est encore noté : à Saint-Antonin-Noble-Val (Tarn-etGaronne), au XIII s., domus est employé avec les deux nombres pour désigner une maison d'habitation comprenant toujours un seul corps de bâtiment ; à Cahors (Lot), les deux nombres désignent une maison d'habitation faite d'un ou peut-être plusieurs bâtiments (Scellès, 1994: I, 157-158).

Sur le territoire de la France métropolitaine, DŎMUS n'aurait pas laissé de trace toponymique selon tous les chercheurs en toponymie qui se sont abstenus... de chercher. La toponymie ne saurait se limiter aux seuls noms de communes et aux anciennes paroisses ou hameaux n'ayant pu accéder ou conserver le titre de commune. La microtoponymie donne la clef de la macrotoponymie : en règle générale, un macrotoponyme actuel (nom de commune) est le nom d'un lieu habité dont le nombre d'habitants n'a cessé de croître jusqu'au XVIII ${ }^{\mathrm{e}}$ s., avec, en règle générale, une église paroissiale. Le lieu actuel, désigné par un microtoponyme, peut avoir été habité jusqu'à devenir chef-lieu de pa- 
roisse, puis avoir subi la désertification jusqu'à devenir simple nom de parcelle.

Dans la microtoponymie de la France métropolitaine, la première syllabe Dom- est le plus fréquente en Bretagne, Basse et Haute. Des noms qui, en Bretagne, pourraient apparemment provenir de DŎMUS, trois séries doivent être écartées :

1. D'une part, les noms issus du vieux breton DOM "profond" (< brittonique DUMNO-), tel Domporz à Crozon (Finistère), attesté Domporz en 1426 (Torchet, 2001: 77). Celui-ci est formé sur dom porz qui signifie "crique profonde" (Deshayes, 2003: 195 et 592).

2. D'autre part, les noms de paroisses issus du latin médiéval DOMINUS "saint" suivi d'un hagionyme : Domalain, commune (Illeet-Vilaine), attesté parrochia de Domalan en 1167, avec une église dédiée à saint Melaine (Bossard, 1930).

3. Enfin, les noms issus du vieux breton DOM "dom" (< latin DŎMĬNUS) (Lagadeuc, 1499: 83), qui s'applique aussi bien à des abbés qu'à des prêtres séculiers ou des sacristains au XV $\mathrm{X}^{\mathrm{e}} \mathrm{s}$. Il apparaît précédant un nom de personne, dans les types de structure suivants :

3.1 DOM + NP : Domhéry, hameau, à Guérande (Loire-Atlantique) : village de dom Henry Lostic, 1646 ; vilage de Dom Hery, 1720 ; Domhery, c.1790 (Buron, 2014). D'après une communication personnelle de G. Buron, dom Henry Lostis est attesté comme prêtre et chapelain dans des aveux locaux entre 1572 et 1576, date de son décès. C'est donc quelques décennies plus tard que le hameau a ainsi été nommé. Ce type de formation continue d'être employé en d'autres lieux de Bretagne au cours du XVII ${ }^{\mathrm{e}} \mathrm{s}$. Il privilégie presque toujours le seul nom de baptême sur le seul nom de famille, le syntagme nom de baptême + nom de famille étant manifestement exclu de l'usage. D'autre part, l'usage de l'article pour déterminer le nom de lieu est aussi exclu.

3.2 lex. + DOM + NP : La Ville Donjean, écart, à Muel (Ille-etVilaine) : La Ville Dom Jeam, 1541 (Bossard, 1930) ; La Ville Donjean, après 1789 (Cassini). Ce type toponymique 
ne semble pas, à défaut d'attestations plus anciennes, antérieur au $\mathrm{XV}^{\mathrm{e}} \mathrm{s}$.

Il est cependant trois exemples médiévaux émanant de Bretagne où domus fonctionne en emploi incontestablement toponymique :

1. En 1243, Henri fils de Robert Symon est réputé propriétaire de quadam domo sita in castello Sancti Chorentini que vocatur Domus filii Bidel (Peyron, 1909: n61). La maison est donc située dans le bourg fortifié de Saint-Corentin à Quimper (Finistère).

2. En 1281, le chevalier Eudes Boucher et sa femme Catherine donnent à l'abbaye de La Joie tous leurs biens situés notamment in villa que dicitur Domus filii Cervi, in parrochia de Ploezoe, in feodo nostro (Rosenzweig, 1895: n³89). Cet ancien écart rural, aujourd'hui disparu, se situait dans la paroisse de Plouay (Morbihan).

3. En 1426, la réformation de la paroisse de La Prénessaye (Côtesdu-Nord) mentionne la présence de six foyers habitant l'écart alors appelé Domlequen (Lorant \& Floury, 2003: 554), aujourd'hui Doulcan. Ce nom est formé de DOMUS + NP LE QUEN ( $<$ bret. KEN "beau, bon", attesté quen au XVe s., cf. Deshayes, 2003: 383).

L'évolution phonétique bien affirmée dans cet exemple, comparée à la stagnation graphico-phonétique particulièrement remarquable dans l'ensemble du corpus toponymique breton (types : Dom Guillaume à La Bouillie, Le Domichel à Lancieux, La Ville Donguy à Plélo, tous dans le département des Côtes-du-Nord), laisse estimer que ces types où dom précède un nom de baptême ne sont pas formés sur dom < DŎMUS "maison d'habitation ; village", mais sur dom < DŎMĬNUS "dom".

En dehors des trois exemples médiévaux assurés, DŎMUS a aussi été utilisé, en Bretagne, pour former des simples du type : Le Don, écart de Peillac (Morbihan) ; et de nombreux dérivés, parmi lesquels les plus anciennement attestés sont : juxta calciatam de Domon en 1152-1200 (Anger, 1911: ${ }^{\circ}$ CCXXVIII) à Saint-Marc-le-Blanc (Ille-et-Vilaine), et medietaria... Dometam au XII ${ }^{\mathrm{e}}$ s. (Bossard, 1930) à Acigné (Ille-etVilaine). 
En dehors de la Haute-Bretagne, l'histoire de DŎMUS présente plusieurs évolutions dans la Galloromania.

La plus récente est présente dans les sources médiolatines, où Domus sert à traduire en contexte latin des toponymes français ou occitans : pour français Maison, dans apud Curtam Domum... de Curmesun vers 1100, aujourd'hui Courmaison à Noyen dans la Sarthe (Vallée, 1950: 290-291) ; pour occitan Mas, dans Domos Chaysias en 1344 devenu Le Mas des Chays en 1539, aujourd'hui Le Mas-des-Chaix dans les Hautes-Alpes (Roman, 1884: 92). En-dehors, notons aussi la traduction de l'alsacien Hus, dans Dietrico et Johanni Virico fratribus de Domo en 1300, déjà die burg ze Husenburg en 1250, aujourd'hui Husenburg à Lautenbach-Zell dans le Haut-Rhin (Stoffel, 1876: 267).

Au rebours, l'exemple toponymique le plus ancien de Domus figure dans le testament du diacre Adalgisel Grimo, éduqué à Verdun (Meuse) et qui fit carrière dans les pays entre la Moselle et le Rhin, texte rédigé en 634 : locum vero cognominante Domo et castrum Teulegio sectum in Vosago, ubi pro Dei reverentia loca sanctorum aedificavi... ipsa loca sancta in predicto loco Doma aut Toleio (Levison, 1932: 79 et 82). Le lieu ainsi appelé désigne la jeune abbaye fondée au pied du castrum, dans la Sarre, domus étant utilisé au sens de "domus Dei"; le toponyme disparaît ensuite de la documentation. Cet exemple est aussi le premier à présenter une variation du genre et de déclinaison de domus (Domo pour masculin de domus, Doma pour féminin), l'année même où la Vita Eligii Noviomagensis fournit le plus ancien exemple lexical de variation du genre sans changement de déclinaison. Deux sources de type différent, écrites à des centaines de kilomètres de distance, preuves de l'évolution de la langue à l'époque mérovingienne... Il faudra attendre longtemps, le $\mathrm{XV}^{\mathrm{e}} \mathrm{s}$., pour qu'apparaisse une autre attestation de domus sous forme simple mais avec changement de déclinaison : borio de Doma alias de Monte Ardito en 1450 (Gourgues, 1873: 99), aujourd'hui Dome à Agonac (Dordogne).

Plus tard, à l'époque carolingienne, domus sert à désigner différents établissements et réussit à se maintenir jusqu'aujourd'hui dans la toponymie, toujours en composition avec un adjectif et seulement dans les zones les plus méridionales du royaume. À Rodès (Pyrénées-Orientales), la chapelle appelée Domonova en 942, devenue hermita de Nostre Senyora de Domanova en 1688 (Ponsich, 1980: 119), aujourd'hui Domanova : chapelle puis prieuré dédié à Notre-Dame. À Tuchan 
(Aude), le village dit villa quae dicitur Domnova en 1106 (Sabarthès, 1912: 117), Donnove en 1706 (Statuts, 1706: CVIII), aujourd'hui Donneuve : prieuré dédié à saint Barthélemy. À Montlaur (Aude), le village dit villa que vocatur Domonova cum servos et ancillas totum et ab integrum en 876 (Devic \& Vaissète, 1875: Preuves, n¹90) devenu Domnove en 1503 (Sabarthès, 1912: 118), aujourd'hui Domneuve : prieuré dédié à saint Julien. À Villeneuve-lès-Maguelone (Hérault), terminio de Domenova vers 1200 (Hamlin, 2000: 138), aujourd'hui Domenoves : il est légitime d'y voir le nom d'un ancien établissement religieux sis aux portes du domaine cujus vocabulum est Villanova que le roi Louis le Débonnaire restitua à l'évêque de Maguelone en 819 (Rouquette \& Villemagne, 1912: nII), établissement qui aurait précédé le diplôme royal ; qui plus est, des découvertes archéologiques nous apprennent que le site, occupé dès l'époque gauloise, s'est développé à partir de l'Antiquité tardive jusqu'au VIII' s. (Vial, 2003: 386). À Châteauvert (Vaucluse), l'alleu épiscopal in villa quae dicitur Domo novo en 952-58 devenu in Domanova en 976 (Didier et alii, 1967: nXIX et XXVI), aujourd'hui Saint-Symphorien : sanctuaire construit entre 955 et 975 et dédié à saint Symphorien (Barruol, 1965: 156). Notons que la source provençale apporte un exemple de l'alternance de genre et de changement de déclinaison de domus.

De même qu'en Bretagne, les formes suffixées galloromanes n'apparaissent qu'après l'an mil en toponymie seule. La plus ancienne figure dans le polyptyque de Saint-Pierre-le-Vif de Sens, aussi appelé « charte de Clovis », faux diplôme rédigé par l'abbaye dans les années 106878 : in villa Domisco (Rouche, 1993: 111), viris religiosis in domo de Domisco Deo servientibus en 1145 (Samarthan, 1720: Instr., col.217), aujourd'hui Doumis à Chalvignac (Cantal). La seconde source nous apprend qu'il s'agit d'un prieuré, situé à quelques centaines de mètres de la Dordogne qui sépare alors les diocèses de Clermont et de Limoges : là se trouvait le site primitif de l'abbaye d'Obazine, transférée audit Obazine (Corrèze) dès 1145 (Barrière, 1998: 202). Il pourrait s'agir, à la base, de la fondation d'une villa et d'un prieuré par l'abbaye de SaintPierre-le-Vif, le suffixe -ISCU s'appliquant à nommer la villa dépendant de la domus Dei. De formation plus tardive est Domaison à Saugues (Haute-Loire), attesté Domezo en 1327 (Chassaing, 1907: 107).

Sauf erreur d'interprétation, les suffixes sont les suivants (nous ne citons qu'un seul exemple par type) : 


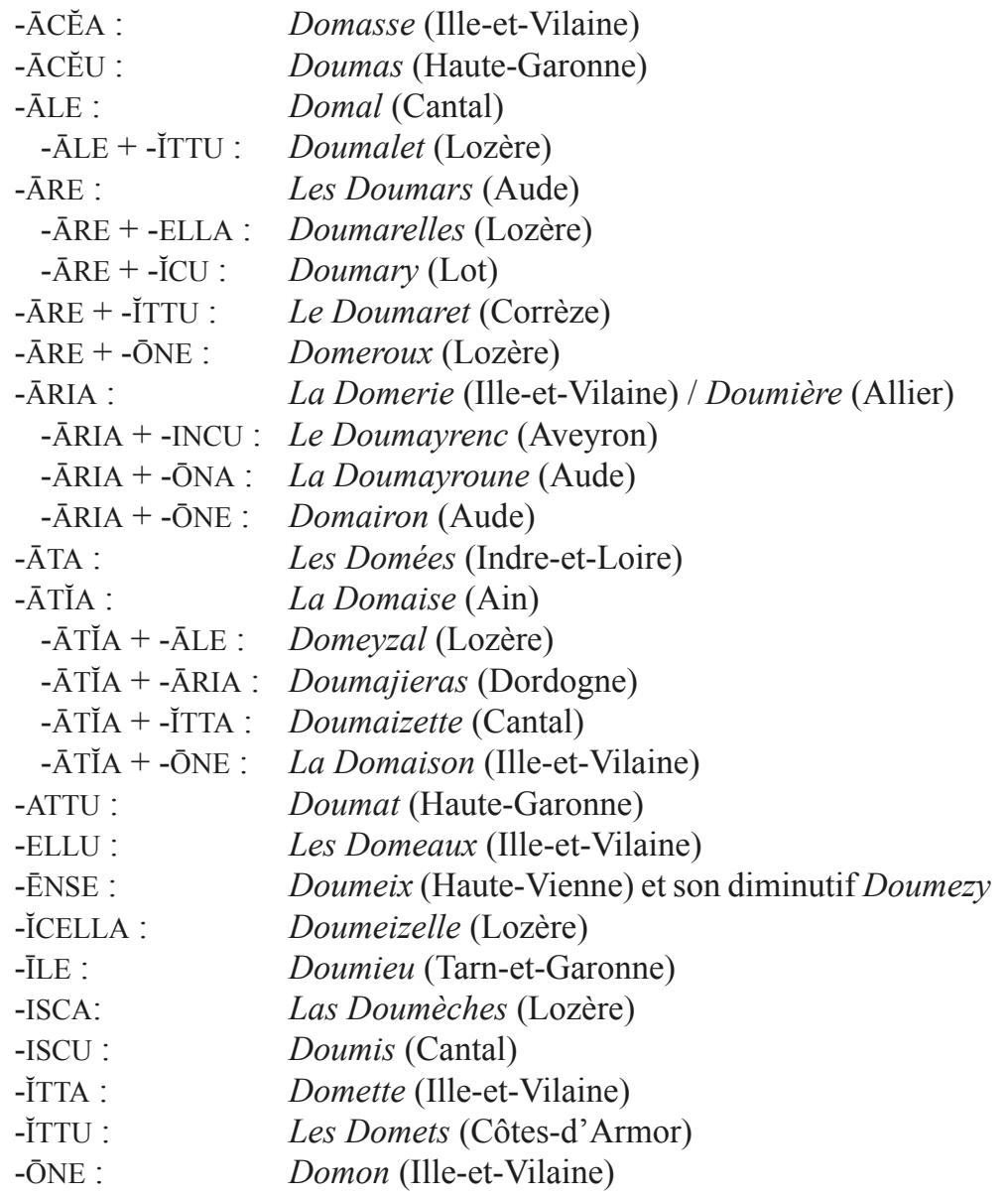

Parmi tous ces dérivés, aucun ne semble provenir d'un nom de famille ou du lexique. Les dérivés en -ĀRǏA ne peuvent être déclarés issus de français moderne domerie "espèce de bénéfice qui n'est ni chapelle, ni prébende, ni prieuré, ni abbaye, \& qui est gouverné par un ecclésiastique qui prend la qualité de dom" (Trévoux, 1752: col.902) ; ce mot a toujours été appliqué à l'Hôpital d'Aubrac et, par extension, aux établissements qu'il a créés. En 1596 le bénéfice concernant l'Hôpital d'Aubrac est nommé le Dom d'Aubrac par Marguerite de Valois (Guessard, 1842: 324) ; en 1598 sa dompnerie et monastere de Nostre Dame d'Aubrac (Ducaunès-Duval, 1877: ${ }^{\circ} \mathrm{CLXIX)}$; en 1668 la domerie d'Aubrac en Rouergue (Callen, 1884: 354). En 1728, Domerie est traité 
comme un nom de lieu, à l'instar de Locdieu, Bonnecombe et Bonneval, trois abbayes au diocèse de Rodez (Almanach, 1728: 38). L'origine du mot est la suivante : mlat. (DŎMĬNUS $>$ ) DOMPNUS + -ĀRǏA $>$ dompnerie $>$ domerie.

En résumé, l'emploi toponymique de DŎMUS se marque par :

1. Le sémantisme religieux de "prieuré (ou chapelle)" dans les formes composées avec un adjectif, ainsi que dans le dérivé DOMISCU "dépendance de prieuré".

2. Le sémantisme de "maison; village" dans toutes les formes simples et suffixées, ainsi que la forme bretonne composée avec un nom de personne ; le fait que 38\% des lieux ainsi désignés soient encore des écarts aujourd'hui (un ou quelques bâtiments habités) plaide en faveur des sens plus précis de "maison; hameau", le hameau désignant une concentration d'habitats sans église, contrairement au village.

3. Le genre masculin en Bretagne et Maine, féminin partout ailleurs.

4. La forme simple principalement dans une bande traversant la France du Nord-Ouest au Sud-Est (Bretagne, Maine, Charente, Périgord, Bourbonnais, Bugey, Gapençais).

5. La forme composée avec un adjectif dans la seule partie méridionale de la France (Pyrénées-Orientales, Aude, Hérault, Vaucluse).

6. La forme composée avec un nom de personne en Bretagne seule (Côtes-d'Armor).

7. La forme suffixée partout en France sauf un grand quart Nord-Est et la Gascogne.

Une telle répartition ne correspond à aucune autre connue. L'emploi toponymique de DŎMUS dans la longue durée (VII $-\mathrm{XIII}^{\mathrm{e}} \mathrm{s}$. au moins, quand il ne s'agit pas de simple traduction) ne permet pas de conclure à de quelconques influences linguistiques de substrat ou de superstrat. D'autre part, son double sémantisme ("prieuré (ou chapelle)" et "maison ; hameau") et son usage dans le temps identiques à ceux de lat. CASA (cf. les monastères appelés La Case-Dieu, La Chaise-Dieu...), incite à cartographier les toponymes issus de CASA : parce qu'extrêmement nombreux, nous avons mis sur carte seulement ceux issus des 
formes simples et syntagmatiques (cinq fois plus nombreux que tous les toponymes issus de DŎMUS, simples, composés et dérivés).

La comparaison des deux cartes est édifiante :

- DŎMUS et CASA sont ignorés dans la Bretagne bretonnante (mis à part deux attestations médiévales de DOMUS), inconnus en Haute-Normandie, Picardie, Lorraine, Bourgogne septentrionale, Franche-Comté, rarissimes à l'est du Rhône ;

- CASA apparaît en faible nombre en Île-de-France, Champagne, en grand nombre en Gascogne.

Les zones identiques d'absence de ces noms résultent très probablement d'un motif commun que seule la cartographie de toponymes issus de centaine d'étymons permettra de connaître.

\section{Bibliographie}

Albanès, Joseph Hyacinthe \& Chevalier, Ulysse. 1901. Gallia christiana novissima. III. Arles. Valence: Imprimerie valentinoise.

Almanach. 1728. Almanach royal pour l'an bissextil MDCCXXVIII calculé au meridien de Paris.... Paris: Veuve d'Houry.

Anger, Pierre. 1911. Cartulaire de l'abbaye de Saint-Sulpice-la-Forêt (Ille-etVilaine). Rennes: Imprimerie Prost.

Barrière, Bernadette. 1998. Moines en Limousin: L'aventure cistercienne. Limoges: PULIM.

Barruol, Jean. 1965. "L'inscription médiévale du prieuré de Saint-Symphorien, près d'Apt". Provence historique 15: 147-157.

Blaise, Albert. 1954. Dictionnaire latin-français des auteurs chrétiens. Turnhout: Brepols.

Böhringer, Letha. 1992. Hinkmar von Reims, De divortio Lotharii regis et Theutbergae reginae. In: MGH, Concilia, IV, Suppl. I. Hannover: Hahn.

Bossard, Pierre. 1930. Dictionnaire topographique du département d'Ille-etVilaine. Rennes: ms.

Buron, Gildas. 2014. Domhéry. Guérande: ms.

Callen, Jules. 1884. L'église métropolitaine et primatiale Sainct André de Bourdeaux [1668]. II. Bordeaux: Féret et fils.

Cassini, César-François. 1756-après 1790. Carte générale de la France. Paris: L. Capitaine.

Chassaing, Augustin. 1907. Dictionnaire topographique du département de la Haute-Loire. Paris: Imprimerie nationale. 
Coromines, Joan. 1995. Onomasticon Cataloniae. IV. Barcelona: Curial Ediciones.

De Poerck, Guy. 1963. "Les plus anciens textes de la langue française comme témoins de l'époque". Revue de linguistique romane XXVII: 1-34.

Deshayes, Albert. 2003. Dictionnaire étymologique du breton. Douarnenez: Chasse-Marée.

Devic, Claude \& Vaissète, Joseph. 1875. Histoire générale de Languedoc. II. Toulouse: Privat.

Didier, Noël ; Dubled, Henri \& Barruol, Jean. 1967. Cartulaire de l'église d'Apt (835-1130 ?). Paris: Librairie Dalloz.

Ducaunès-Duval, Ariste. 1877. Archives historiques de la Gironde. XVII. Bordeaux: Charles Lefebvre.

Eckhardt, Karl August. 1969. Lex salica. In: MGH, Leges nationum Germanicarum, IV/2. Hannover: Hahn.

Fawtier, Robert. 1925. Ingomar, historien breton. Dans Halphen, Louis \& Fawtier, Robert (éds). Mélanges d'histoire du Moyen Âge offerts à $M$. Ferdinand Lot. Paris: Librairie Champion, 181-203.

Feuillet de Conches, Félix. 1855. Journal du marquis de Dangeau. V. Paris: Firmin Didot frères.

Flobert, Pierre. 2002. La vie ancienne de saint Samson de Dol. Paris: CNRS.

Gasca Queirazza, Giuliano ; Marcato, Carla ; Pellegrini, Gian Battista ; Petracco Sicardi, Giulia \& Rossebastiano, Alda. 2006. Dizionario di toponomastica: Storia e significato dei nomi geografici italiani. Torino: UTET.

Glare, Peter G.W. 2012. Oxford Latin Dictionary. Oxford: Oxford University Press.

Gourgues, Alexis de. 1873. Dictionnaire topographique du département de la Dordogne. Paris: Imprimerie nationale.

Guessard, François. 1842. Mémoires et lettres de Marguerite de Valois. Paris: Jules Renouard et $C^{\text {ie }}$.

Hamlin, Frank R. 2000. Toponymie de l'Hérault: Dictionnaire topographique et étymologique. Millau: Éditions du Beffroi.

Heinzelmann, Martin. 1993. Villa d'après les œuvres de Grégoire de Tours. Dans Magnou-Nortier, Élisabeth (éd.). Aux sources de la gestion publique. I. Enquête lexicographique sur fundus, villa, domus, mansus. Lille: Presses Universitaires de Lille, 45-70.

Hilty, Gerold. 1997. La naissance du français. I. Le latin et la fragmentation de la Romania. Dans Blampain, Daniel ; Goose, André ; Klinkenberg, Jean-Marie \& Wilmet, Marc. Le français en Belgique: une communauté, une langue. Bruxelles: Duculot, 59-76. 
Hummel, Pascale. 2004. La maison et le chemin: Petit essai de philologie théologique. Berne: Peter Lang.

Jones, Michael. 2001. The naming of parts : remarques sur le vocabulaire des résidences seigneuriales et princières en Bretagne au Moyen Âge. Dans Renoux, Annie (éd.). Aux marches du palais. Qu'est-ce qu'un palais médiéval ? Données historiques et archéologiques: Actes du VII Congrès international d'archéologie médiévale (Le Mans, 9-11 septembre 1999). Caen/Le Mans: Société d'archéologie médiévale/Publications du LHAM, 45-54.

Krusch, Bruno. 1885. Gregorii episcopi Turonensis Liber in gloria confessorum. Dans MGH, SRM, I/2. Hannover: Hahn, 294-370.

Krusch, Bruno. 1885. Gregorii episcopi Turonensis Liber in gloria martyrum. Dans MGH, SRM, I/2. Hannover: Hahn, 34-111.

Krusch, Bruno. 1902. Vita Eligii Noviomagensis. Dans MGH, SRM, IV. Hannover: Hahn.

Krusch, Bruno \& Levison, Wilhelm. 1951. Gregorii episcopi Turonensis Historiarum libri X. Dans MGH, SRM, I/1. Hannover: Hahn.

Krusch, Bruno \& Levison, Wilhelm. 1913. Vita Wilfridi episcopi Eboracensis. Dans MGH, SRM, VI. Hannover: Hahn.

La Borderie, Arthur de. 1885. "Recueil d'actes inédits des ducs et princes de Bretagne". Bulletin et mémoires de la Société archéologique d'Ille-etVilaine XVII: 341-436.

Lagadeuc, Jehan. 1499. Catholicon. Tréguier: Jehan Calvez.

Le Men, René-François-Laurent \& Ernault, Émile. 1886. Cartulaire de Landévennec. Dans Mélanges historiques. Choix de documents. Paris: Imprimerie nationale, V, 533-600.

Levison, Wilhelm. 1932. "Das Testament des Diakons Adalgisel-Grimo vom Jahre 634". Trierer Zeitschrift VII: 69-85.

Lorant, Éric \& Floury, Jérôme. 2003. Montres générales \& réformations des fouages de Bretagne aux XVe \& XVI siècles. I. Évêché de Saint-Brieuc. Rennes: Sajef.

Marchot, Paul. 1895. Les Gloses de Cassel : Le plus ancien texte réto-roman. Fribourg: Librairie de l'Université.

Meuret, Jean-Claude. 1993. Peuplement, pouvoir et paysage sur la marche Anjou-Bretagne. Laval: Société d'archéologie et d'histoire de la Mayenne.

Meyer-Lübke, Wilhelm. 1935. Romanisches etymologisches Wörterbuch. Heidelberg: Carl Winter.

Migne, Jacques-Paul. 1850. Patrologia latina. XCI. Venerabilis Bedae. 2. Paris: Migne.

Migne, Jacques-Paul. 1850. Patrologia latina. XCII. Venerabilis Bedae. 3. Paris: Migne. 
Mohrmann, Christine. 1962. "Les dénominations de l'église en tant qu'édifice en grec et en latin au cours des premiers siècles chrétiens". Revue des Sciences religieuses 36: 155-174.

Morice, Hyacinthe. 1742. Mémoires pour servir de preuves à l'Histoire ecclésiastique et civile de Bretagne. I. Paris: Charles Osmont.

Oheix, André. 1913. "Nécrologe de l'abbaye de Landévennec". Bulletin diocésain d'histoire et d'archéologie XIII: 33-49.

Paris, Gaston. 1872. "La Vie de saint Léger. Texte revu sur le ms. de Clermont-Ferrand". Romania I: 273-317.

Pertz, Georg Heinrich. 1835. Capitularia regum Francorum: Supplementa tomi I. Constitutiones regum Germaniae. Dans MGH, Leges, I. Hannover: Hahn.

Peyron, Paul. 1909. Cartulaire de l'église de Quimper. Quimper: A. de Kerangal.

Ponsich, Pierre. 1980. Limits histórics i repertori toponímic dels llocs habitats dels antics paisos de Rosselló, Vallespir, Conflent, Capcir, Cerdanya, Fenolledès. Prades: Terra nostra.

Roman, Joseph. 1884. Dictionnaire topographique du département des Hautes-Alpes. Paris: Imprimerie nationale.

Rosenzweig, Louis. 1895. Cartulaire général du Morbihan. Vannes: Lafolye.

Rouche, Michel. 1993. "Le polyptyque de Saint-Pierre-le-Vif de Sens pour Saint-Pierre de Mauriac : grand domaine ou grand revenu ?". Dans Magnou-Nortier, Élisabeth (éd.). Aux sources de la gestion publique. I. Enquête lexicographique sur fundus, villa, domus, mansus. Lille: Presses Universitaires de Lille, 103-121.

Rouquette, Julien \& Villemagne, Augustin. 1912. Cartulaire de Maguelone. I. Montpellier: Librairie Louis Valat.

Sabarthès, Antoine. 1912. Dictionnaire topographique du département de l'Aude. Paris: Imprimerie nationale.

Samarthan, Denis. 1720. Gallia christiana, in provincias distributa. II. Paris: Typographia regia.

Scellès, Maurice. 1994. Structure urbaine et architecture civile de Cahors aux $X I I^{e}, X I I I^{e}$ et XIV ${ }^{e}$ siècles (Thèse doctorale). Toulouse: Université Toulouse-II - Histoire.

Souter, Alexander. 1949. A Glossary of Later Latin to 600 A.D. Oxford: Clarendon Press.

Statuts. 1706. Statuts synodaux du diocese de Narbonne publiez au synode tenu le XVI. et XVII. Juin M.DCC.VI. Narbonne: G. Besse.

Stoffel, Georg. 1876. Topographisches wörterbuch des Ober-Elsass: Die alten und neuen ortsnamen enthaltend. Mülhausen: Veuve Bader et $\mathrm{C}^{\mathrm{ie}}$. 
Trévoux. 1752. Supplément au Dictionnaire universel françois et latin vulgairement appellé Dictionnaire de Trévoux. I. Paris: Compagnie des libraires associés.

Torchet, Hervé \& Yann. 2001. Réformation des fouages de 1426: Diocèse ou évêché de Cornouaille. Paris: Éditions de La Pérenne.

Vallée, Eugène. 1950. Dictionnaire topographique du département de la Sarthe. I. Paris: Imprimerie nationale.

Vial, Julien. 2003. Carte archéologique de la Gaule: Le Montpelliérais. 34/3. Paris: Académie des Inscriptions et belles-lettres.

Wartburg, Walther von. 1922-2005. Französisches etymologisches wörterbuch (FEW): Eine darstellung des galloromanischen sprachschatzes. BonnLeipzig-Bâle: Teubner-Mohr-Zbinden.

Wolf, Heinz Jürgen. 1998. Toponomastica barbaricina: I nomi di luogo dei comuni di Fonni, Gavoi, Lodine, Mamoiada, Oliena, Ollolai, Olzai, Orgòsolo, Ovodda. Nùoro: Insula.

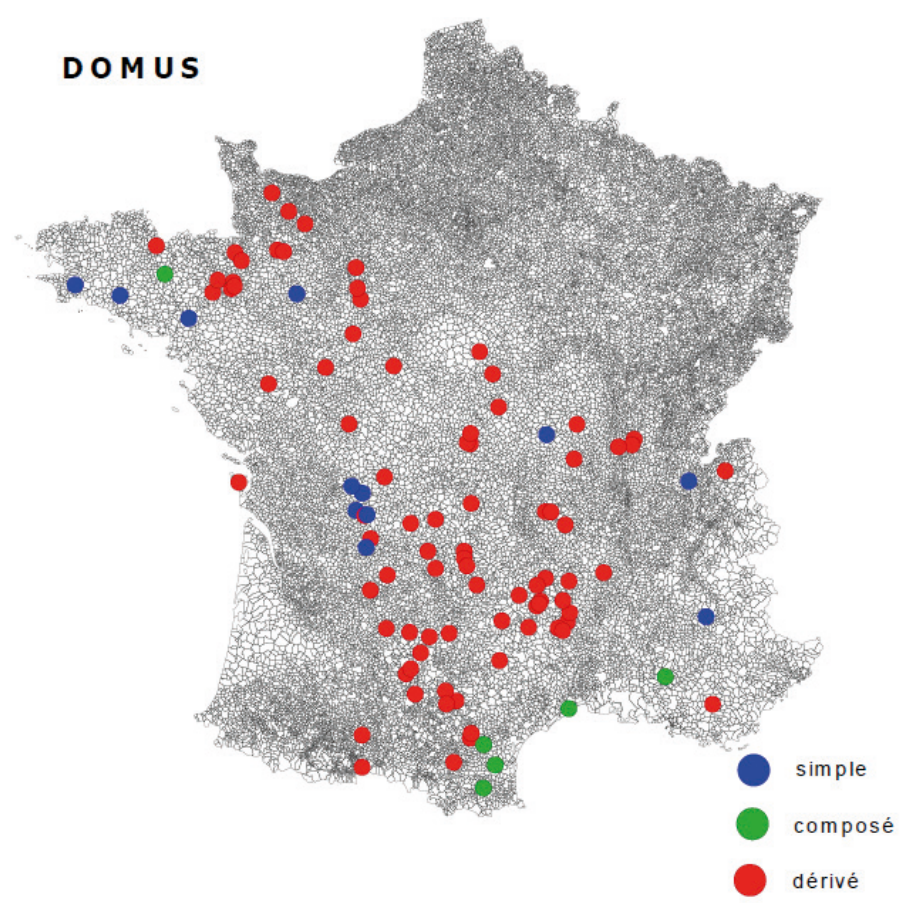




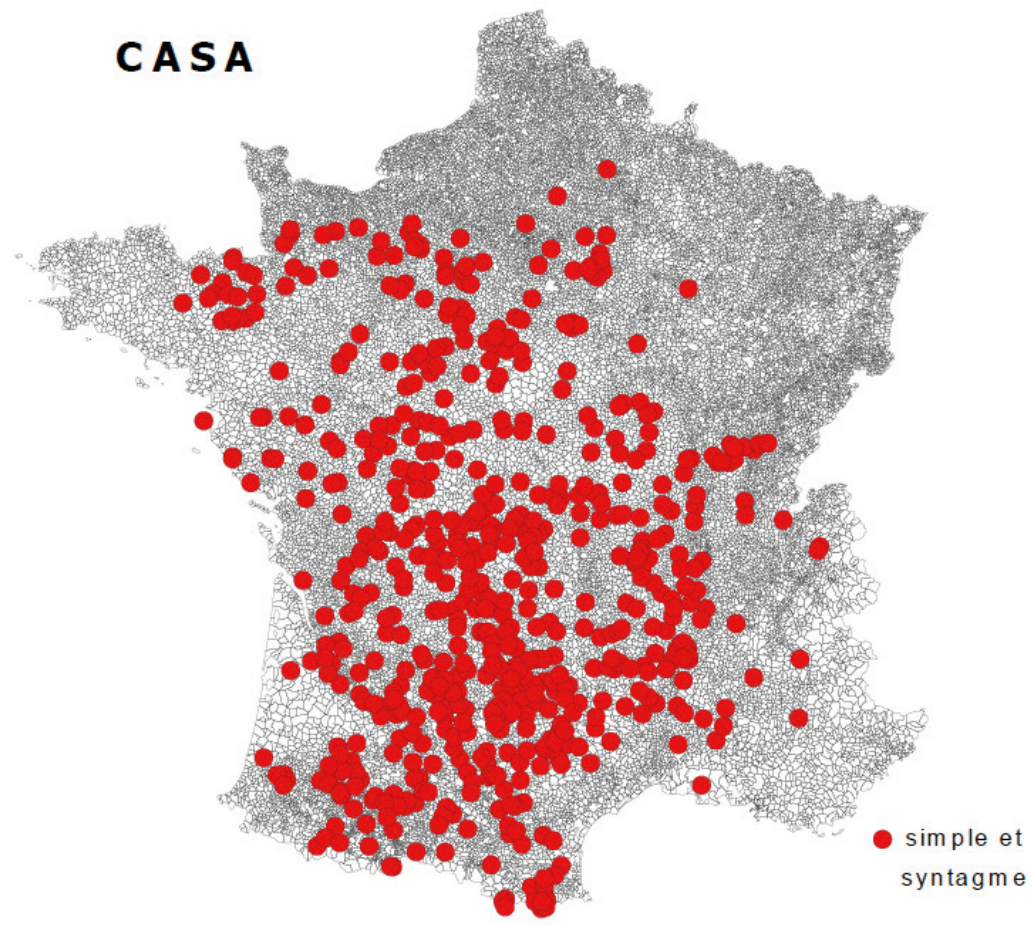


